Grant Report

\title{
The Development of Anxiety in Youth Study (DAYS): A Prospective Study of Trajectories of Brain Maturation among Youth at Risk for Anxiety ${ }^{\dagger}$
}

\author{
Adriana Galván ${ }^{1, *}$, Tara S. Peris ${ }^{2}$ \\ 1 Department of Psychology, University of California, Los Angeles, CA 90024, USA \\ 2 Department of Psychiatry, University of California, Los Angeles, CA 90024, USA \\ $\dagger$ This research was funded by National Institute of Mental Health \\ (R01MH110476-01A1). \\ * Correspondence: Adriana Galván, Email: agalvan@ucla.edu.
}

\begin{abstract}
Anxiety disorders are common across development and are associated with significant functional impairment and distress. Despite neuroimaging advancements in uncovering key brain regions, including the amygdala and prefrontal regions, implicated in anxiety, there is a need to characterize the role of the ventral striatum given its well-documented link to several key anxiety processes. This study aims to characterize specific neural mechanisms that underlie the evolution of illness and impairment using a longitudinal design and to move beyond traditional case control designs to provide a more comprehensive view of the developmental psychopathology of pediatric anxiety.
\end{abstract}

KEYWORDS: anxiety; development; brain; reward

\section{G Open Access}

Received: 10 June 2020

Accepted: 06 December 2020

Published: 16 December 2020

Copyright $\odot 2020$ by the author(s). Licensee Hapres, London, United Kingdom. This is an open access article distributed under the terms and conditions of Creative Commons Attribution 4.0 International License.

\section{INTRODUCTION}

Anxiety disorders occur commonly among children and adolescents [1], and they are associated with substantial distress and impairment [2]. When left untreated, these disorders typically run a chronic, fluctuating course, and they can place youth at risk for a host of subsequent difficulties, including substance use, depression, and educational/occupational underachievement [3-5]. Although evidencebased treatments exist and are helpful to many affected youth, few achieve full remission and relapse is unfortunately common over the longterm [6,7]. Against this backdrop, there remains a need to better understand factors that shape the course and persistence of anxiety and that might be inform novel treatment development.

As an emotion, anxiety captures a diffuse sense of unease or apprehension, often occurring in response to unknown or future-oriented events [8]. It is distinct from fear, which is an in-the-moment response to a known threat. As with all emotions, anxiety is conceptualized as a normal, natural, and necessary mood state. It occurs along a continuum, 
and is cause for concern only at its more problematic extremes (i.e., when marked distress and impairment are present). Thus, the anxiety spectrum includes mild, transient, and normative symptoms at one end and pervasive, severe, and highly impairing symptoms at the other.

Longitudinal studies of youth anxiety reveal substantial heterogeneity in outcome, but with limited exception [9], rarely consider biological substrates that might account for these differences. By contrast efforts to understand the biological underpinnings of anxiety have been almost exclusively cross-sectional. This work pinpoints the amygdala (AMY) and ventrolateral prefrontal cortex (vlPFC) as central hubs in a fear circuit that also includes the anterior cingulate cortex (ACC) [10-12]. In both animal studies and human subject research, atypical responses to threat are reported in these regions both among adolescents with anxiety disorders and among those with a history of behavioral inhibition (BI) [11-15]. Previous studies $[16,17]$ suggest that the vlPFC regulates arousal through its effects on attentional control. Human lesion [18] and brain imaging [19] work also implicate the ventromedial PFC (vmPFC) in regulating AMY activity in individuals with and without trait anxiety. Other research has focused on approach systems, specifically the ventral striatum, finding evidence for heightened striatal sensitivity among adolescents with a history of BI [20-22] and/or current anxiety disorder [23].

Despite these advances, the field lags in two key areas. First, although pediatric anxiety neuroimaging studies have proliferated over the past decade, there has been relatively less attention to the role of the ventral striatum in existing models. This is understandable in light of its central standing in the reward literature. At the same time, it is unfortunate given its well-documented link to several key anxiety processes (e.g., attention bias, fear conditioning, motivation) [24,25]. Indeed, emerging evidence suggests that atypical engagement of the ventral striatum is characteristic of youth with anxiety [26]. Second, the integration of approach and avoidance systems has received limited attention to date. Recent work suggests that both adolescents with generalized anxiety disorder (GAD) [10] and young adults with a childhood history of BI [27] exhibit altered frontostriatal/amygdalostriatal functional connectivity during threat processing. What remains unknown is the developmental trajectory of this unique connectivity phenotype and whether/how it explains worsening of anxiety symptoms and functional impairment as youth move through adolescence.

\section{The Current Study}

The Development of Anxiety in Youth Study (DAYS) is a prospective longitudinal study of youth across the anxiety spectrum. It aims to characterize specific neural mechanisms that underlie the evolution of illness and impairment and to move beyond traditional case control designs to provide a more comprehensive view of the developmental psychopathology of pediatric anxiety. Prior cross-sectional research has 
been crucial for identifying circuitry that underlies pediatric anxiety. Yet, by design, it cannot answer questions about how these nodes develop. Equally important, it has overlooked the role of other systems with known links to anxiety, including the ventral striatum [24]. Finally, by relying on case control designs, it has sacrificed power and compromised the ability to understand individual differences in outcome. DAYS addresses these gaps by focusing on: (1) the interplay of a more complete suite of circuitry in contributing to youth anxiety and (2) the development of this network and its links to anxiety over time. Findings will illuminate the component/interactive processes by which approach, avoidance, and cognitive control circuitry contribute to anxiety. Study procedures for this study include the collection of multivariate and multi-informant data, including brain imaging, cognitive tests, computerized assessments, sleep assessments, clinical interviews and self-reports feelings and behaviors. Together, these rich data will permit a holistic examination of the underlying antecedents, mechanisms and behavioral patterns that will help guide treatment.

\section{A Neurodevelopmental Framework}

Age-expectant and adaptive changes in a distributed network of brain regions underlie behavioral sensitivity to risk and reward among typically-developing (TD) youth [28]. Our work has examined how the interplay of approach, avoidance, and cognitive control circuitry explains the propensity for increased risk-taking during adolescence, revealing that, among TD youth, subcortical regions underlying approach behaviors (e.g., ventral striatum; VS) show greater governance over behavior compared to the prefrontal regions that underlie regulation and inhibition [29-31]. These distinct developmental trajectories account for increases in exploration and risky behavior observed among TD adolescents [32-34]. Here, we use this model as a backdrop for understanding deviations from the TD trajectory among anxious youth. According to recent meta-analyses [35], epidemiological studies [36], and DSMV [37]. Anxiety peaks in adolescence and is characterized by behaviors at odds with normative development (risk-aversion), as well as neural perturbations associated with both approach and avoidance responses [10,12,16,20,23]. As noted, the role of the ventral striatum has been largely overlooked despite its role in processes central to anxiety (e.g., attention bias) [26]. Moreover, there is little understanding of the integrative relationships between approach and avoidant processes and the role that maturing cognitive control circuitry plays in modulating these limbic-based fear systems and influencing outcomes.

\section{AIMS OF THE GRANT}

Aim 1: To model connectivity among approach-, avoidance- and cognitive control circuitry during risky decision-making tasks across a continuum of anxious phenotypes cross-sectionally. We apply Dynamic 
Causal Modeling (DCM) and novel functional connectivity methods [38] to advance current neurobiological models. Through this grant we aim to characterize the relationship between distinct neural nodes through the use of connectivity tools (dynamic causal modeling [DCM] and novel models of hemodynamic response/associations of response trajectories across regions). DCM is a powerful tool used to infer relational architecture of coupled or distributed systems [39]. It measures associations among physically disparate regions, and critically, the direction of those associations. DCM is used with fMRI data to create a simple model of neural dynamics in a network of interacting brain regions [40]. This model estimates how changes in neuronal activity in one node sequentially influence activity in other nodes. Since its inception, DCM has been refined to furnish an explicit generative model of relational linkages underlying observed data. Although it is a powerful tool, DCM is limited in its ability to examine several key attributes of functional connectivity (FC), including peak amplitude and latency and functional canonical correlations (FCC). Peak amplitude and latency correspond to the highest level of activity and shape of the hemodynamic response curve that contributes to connectivity parameters. FCC measures associations between entire activation trajectories for two regions, without focusing on particular features. Both of these classes of measurement are suitable for trial-based experiments, do not assume stationarity of the activation time series observed for each brain region and allow adjustments for other brain regions through partial correlations. These tools have been used with clinical populations [41] and anxious youth [38] can be used to determine whole brain network structure instead of simply doing FC analyses between ROIs, and are flexible, taking brain activity data as inputs but also accepting peripheral physiological measures into the FC function [38]. Specifically, these approaches will allow us to identify the precise contributions of known (AMY, PFC) and novel (VS) regions [39] by testing interactional and directional effects between prefrontal and limbic regions.

Aim 2: To track trajectories of symptoms, behavior and neural activation among youth who vary in anxiety longitudinally. Exploratory Aim: To examine trajectories of connectivity among approach-, avoidanceand cognitive control circuitry in youth who vary on anxiety symptomatology. We will fit DCMs at each time-point and use the resulting parameter estimates in longitudinal models to characterize changes in relational patterns at both the individual and group levels.

\section{PROCEDURES}

Overall Structure. This study includes a community sample $(n=120)$ of youth ages 9-13 years at baseline selected to capture the full range of anxiety with oversampling at the more severe end of the distribution. Efforts are made to oversample at the elevated end of the spectrum using the parent- and self-reported Screen for Child Anxiety Related Disorders 
(SCARED) [42] as a screening tool. Importantly, our specified age range is not meant to capture first emergence of anxiety or, necessarily, the transition to disorder. Rather, it is chosen based on robust evidence documenting that symptoms are likely to worsen in this window. Although prior research in community samples suggests that for some youth, there will be attenuation of anxiety symptoms as they progress toward adolescence, it also highlights marked heterogeneity in outcomes with pathways of persistence and worsening for some [43]. Indeed, the average age of onset for several forms of anxiety disorder (a distinction made based on distress and impairment) is during adolescence, by definition suggesting a worsening of symptoms relative to childhood.

Participants are followed annually for three years to track trajectories of change in approach, avoidance, and cognitive control circuitry along with anxiety symptoms and functional impairment. Measurements are taken at year 1 and then twice more annually. At each time-point, participants perform computer tasks while undergoing fMRI designed to elicit engagement of the circuitry of interest, as well as completing selfreport, behavioral, and psychophysiological measures. The computer tasks have been validated in our prior cross-sectional work [44] and have the distinct advantage of assessing unique and interactive contributions of approach-, avoidance-, and cognitive control circuitry. They position us to understand both the avoidance that characterizes the anxious phenotype and heterogeneity that may emerge over time with respect to risk-taking behavior (e.g., coping via risky behaviors such as drinking). This is particularly important given that a subset of anxious youth will exhibit increases in risk taking behavior over time, including substance use, distracted driving, and unprotected sex [45-51]. Study tasks are complemented by innovative neuroimaging models that specifically test the direction of influence among circuitry underlying these processes [40]. Uncovering the dynamics of this network will elucidate how regions previously studied in isolation interact to contribute to heterogeneity in symptom course and will identify targets for novel intervention.

Research Participants. This study enrolls participants who are (1) ages 9-13 at baseline; (2) right-handed; (3) free of metal; (4) have no medical or psychiatric conditions contraindicating study participation (e.g., suicidality); (5) have no current or lifetime history of treatment for anxiety, or history of taking medication for anxiety. Participants are ineligible for the study if they (1) use current psychotropic medication other than those that can be discontinued safely for brief washout prior to scanning; (2) have current major depressive disorder (MDD) as determined by semi-structured diagnostic interview; (3) present with other serious mental illness (i.e., schizophrenia, substance abuse) as assessed via semi-structured diagnostic interview. We exclude youth for whom naturalistic longitudinal study without intervention would pose medical or safety concerns. This includes youth with psychosis, eating disorder, and active self-injury and/or suicidal ideation. Youth with 
medical conditions that may require ongoing intervention and/or medications that would interfere with imaging are also excluded, in consultation with our study physician.

\section{Assessment}

Symptoms are assessed both dimensionally and categorically using gold standard procedures. In line with our emphasis on capturing the full spectrum of anxiety symptom severity, participants complete the Screen for Child Anxiety Related Disorders (SCARED) [42], a 41-item questionnaire, which correlates strongly with clinician-based measures of symptom severity, provides a psychometrically-sound dimensional measure across the anxiety continuum, and enhances our ability to observe the evolution of symptoms over time.

To capture potential anxiety disorder diagnosis as well as relevant comorbidities, the Anxiety Disorders Interview Schedule for DSM-IV, Child Version (ADIS-C) [52] is also administered by trained evaluators. The ADIS assesses anxiety, mood, and externalizing behavior disorders in youth and screens for the presence of several additional disorders including developmental, psychotic, and somatoform disorders. Additionally, it provides information regarding age of onset, impairment, and avoidance [53]. The interview is both reliable and valid [54].

Additional domains of measurement capture potential risk and protective factors and include variables such as life stress, family functioning, peer relationships, puberty, and sleep.

\section{Brain Imaging Tasks}

Broadly, the neuroimaging tasks in this study are designed to measure risk-taking and striatal-based learning. The former is measured using the "Driving Game". In this task, participants move a car along a computerized track with the goal of reaching the end as fast as possible to maximize earnings. Traffic lights positioned at intersections turn yellow as the car approaches. Drivers choose whether to make the cautious decision and stop at the light (adding a short delay) or make a risky decision and drive through the intersection to reach the finish line (gaining time and earnings). However, if the light turns red while the participant is crossing the intersection, the car crashes and there is a longer delay. There are two 12-min rounds, each using a track with 20 intersections, treated as separate trials with a jittered inter-trial interval (ITI). The latter is measured via a learning task called the "T-Shirt Game". Participants are asked to learn associations between t-shirt patterns and universities. They will be presented with one t-shirt pattern (e.g., swirls) that is paired with a university (e.g., Northern University) on $80 \%$ of trials. They press a button when they see the correct pair. 


\section{Laboratory Tasks}

Participants complete three computer tasks that capture information processing domains expected to interact with our risk taking measures of interest. These include measures of interpretation bias, decision-making, and frustration tolerance. Biased appraisals are captured via the Performance-Based Interpretation Bias Task [55]. This task is a lab measure of how youth attribute threat to environmental ambiguity, and it requires participants to watch a screen as a word appears (500 ms), with a sentence following the word and then indicate via button press whether the word and sentence are related ( $n=240$ trials). The program provides the percentage of neutral/threat words endorsed as relevant to ambiguous sentences, and reaction time (RT). This task has established psychometric properties and predicts a significant proportion of variance in anxiety symptoms in our prior work. ( 15 min). Participants also complete the Cups Task, which measures decision-making under conditions of potential gains and losses [56]. In both gain and loss domains, participants are asked to choose between a certain and uncertain option. Depending on the domain, the certain option is to win or lose $\$ 2$ for sure, whereas the uncertain (risky) option consists of a probability $(0.20,0.33$, or 0.50$)$ of a larger win ( $\$ 4, \$ 6$, or $\$ 10$ ) or no win in the gain domain or of a greater loss ( $\$ 4, \$ 6$, or $\$ 10$ ) or no loss in the loss domain. This task uses images of overturned cups divided by a vertical line that demarcates which side of the line is associated with certain and uncertain monetary outcomes. Participants are asked to choose between the certain and uncertain option. After the choice is made, the gamble is resolved immediately, allowing participants to experience the consequences of the gains or losses. Outcome probabilities and the amount of money in this task are manipulated in such a way that the overall expected value of the certain and uncertain outcome is equal. Thus, this task examines whether or not participants' decisions are influenced by who they're making decisions for and/or whether the choice is presented as a gain or a loss. Participants perform 2 runs of this task, each run is approximately 7 min long. ( 14 min). The final laboratory task is the Mirror-Tracing Persistence TaskComputerized Version (MTPC-C, $15 \mathrm{~min}$ ). The computerized Mirror Tracing Persistence task has been shown to be difficult and frustrating [57] and has been used in the literature as a behavioral measure of distress tolerance in adolescents [58]. Participants are to move a red dot along lines of a star presented on a computer monitor with a computer mouse. The mouse is programmed to move the red dot in the opposite direction of physical movement of the mouse. In this way, the task simulates tracing an object that is viewed in a mirror. There are 3 levels used to increase stress and frustration, followed by a final level where participants can discontinue the task by pressing a key on the keyboard. Distress tolerance is measured as time to task termination on this final level. Using the Positive and Negative Affect Scale (PANAS), an established measure of 
positive and negative affect, youth rate their levels of positive and negative affect before and after task-completion ( 15 min).

\section{Biological Variables}

Physiological data is collected during the Mirror-Tracing and Cups tasks. These variables will be use as covariates in analyses to determine individual differences in physiological response as related to behavior and anxiety.The primary outcomes obtained during the MTPT-C include autonomic reactivity (galvanic skin response; GSR) and startle (electromyography; EMG). GSR is continuously recorded from two electrodes positioned on the distal phalanges of the fore- and middle fingers of the non-dominant hand. Startle is collected using orbicularis oculi EMG recorded continuously from two electrodes below each eyelid and a ground electrode behind the ear. Electrodes are filled with conduction gel as contact medium between skin and electrode.

\section{CONCLUSIONS}

We report on the DAYS Project, which is currently completing data collection. Findings from this study have the potential to inform our understanding of how anxiety develops over time and what contributes to risk of developing anxiety. In turn, the hope is that they might inform the development of novel interventions. Although speculative at this juncture, we see several potential applications. The longitudinal design will allow us to examine changes across a period of heightened plasticity to determine whether there are developmental windows during which we can leverage change to introduce positive remodeling (e.g., novel therapeutics). New interventions might directly target identified biomarkers (e.g., neurocognitive therapies to normalize aberrations in avoidance circuitry). Alternatively, findings may be used to make existing psychosocial interventions more potent and efficient. For example, given that cognitive behavior therapy (CBT) involves a collection of treatment techniques, awareness of where deficits lie (e.g., compromised cognitive control systems) may point to treatment components that require greater emphasis (e.g., cognitive coping skills). Finally, we note that while direct clinical translation remains steps away, the rich multivariate dataset that emerges from this project will allow testing of profiles at multiple levels of analysis and their role in anxiety at the individual level.

\section{AUTHOR CONTRIBUTIONS}

AG and TP wrote the manuscript.

\section{CONFLICTS OF INTEREST}

The authors declare that they have no conflicts of interest. 


\section{FUNDING}

This project is funded by the National Institute of Mental Health (R01MH110476-01A1).

\section{ACKNOWLEDGMENTS}

The authors wish to thank the research team and research participants.

\section{REFERENCES}

1. Costello EJ, Mustillo S, Erkanli A, Keeler G, Angold A. Prevalence and development of psychiatric disorders in childhood and adolescence. Arch Gen Psychiatry. 2003;60(8):837-44. doi: 10.1001/archpsyc.60.8.837

2. Essau CA, Lewinsohn PM, Olaya B, Seeley JR. Anxiety disorders in adolescents and psychosocial outcomes at age 30. J Affect Disord. 2014;163:125-32. doi: 10.1016/j.jad.2013.12.033

3. Bardone AM, Moffitt TE, Caspi A, Dickson N, Stanton WR, Silva PA. Adult physical health outcomes of adolescent girls with conduct disorder, depression, and anxiety. J Am Acad Child Adolesc Psychiatry. 1998;37(6):594601. doi: 10.1097/00004583-199806000-00009

4. Woodward LJ, Fergusson DM. Life course outcomes of young people with anxiety disorders in adolescence. J Am Acad Child Adolesc Psychiatry. 2001;40(9):1086-93. doi: 10.1097/00004583-200109000-00018

5. Wehry AM, Beesdo-Baum K, Hennelly MM, Connolly SD, Strawn JR. Assessment and Treatment of Anxiety Disorders in Children and Adolescents. Curr Psychiatry Rep. 2015;17(7):591. doi: 10.1007/s11920-015-0591-z

6. Ginsburg GS, Becker EM, Keeton CP, Sakolsky D, Piacentini J, Albano AM, et al. Naturalistic follow-up of youths treated for pediatric anxiety disorders. JAMA Psychiatry. 2014;71(3):310-8. doi: 10.1001/jamapsychiatry.2013.4186

7. Piacentini J, Bennett S, Compton SN, Kendall PC, Birmaher B, Albano AM, et al. 24- and 36-week outcomes for the child/adolescent anxiety multimodal study (CAMS). J Am Acad Child Adolesc Psychiatry. 2014;53(3):297-310. doi: 10.1016/j.jaac.2013.11.010

8. Sadock BJ, Sadock VA, Ruiz P. Kaplan and Sadock's Synopsis of Psychiatry: Behavioral Sciences/Clinical Psychiatry. 11th ed. New York (US): Lippincott Williams \& Wilkins; 2013.

9. White LK, Degnan KA, Henderson HA, Pérez-Edgar K, Walker OL, Shechner T, et al. Developmental Relations Among Behavioral Inhibition, Anxiety, and Attention Biases to Threat and Positive Information. Child Dev. 2017;88(1):141-55. doi: 10.1111/cdev.12696

10. Monk CS, Telzer EH, Mogg K, Bradley BP, Mai X, Louro HMC, et al. Amygdala and ventrolateral prefrontal cortex activation to masked angry faces in children and adolescents with generalized anxiety disorder. Arch Gen Psychiatry. 2008;65:568-76. doi: 10.1001/archpsyc.65.5.568

11. Monk CS, Nelson EE, McClure EB, Mogg K, Bradley BP, Leibenluft E, et al. Ventrolateral prefrontal cortex activation and attentional bias in response to angry faces in adolescents with generalized anxiety disorder. Am J Psychiatry. 
2006;163:1091-7. doi: 10.1176/ajp.2006.163.6.1091

12. Britton JC, Bar-Haim Y, Carver FW, Holroyd T, Norcross MA, Detloff A, et al. Isolating neural components of threat bias in pediatric anxiety. J Child Psychol Psychiatry Allied Discip. 2012;53:678-86. doi: 10.1111/j.14697610.2011.02503.x

13. Pérez-Edgar K, Roberson-Nay R, Hardin MG, Poeth K, Guyer AE, Nelson EE, et al. Attention alters neural responses to evocative faces in behaviorally inhibited adolescents. Neuroimage. 2007;35:1538-46. doi: 10.1016/j.neuroimage.2007.02.006

14. Guyer AE, Lau JYF, McClure-Tone EB, Parrish J, Shiffrin ND, Reynolds RC, et al. Amygdala and ventrolateral prefrontal cortex function during anticipated peer evaluation in pediatric social anxiety. Arch Gen Psychiatry. 2008;65:1303-12. doi: 10.1001/archpsyc.65.11.1303

15. Beesdo K, Lau JYF, Guyer AE, McClure-Tone EB, Monk CS, Nelson EE, et al. Common and distinct amygdala-function perturbations in depressed vs anxious adolescents. Arch Gen Psychiatry. 2009;66:275-85. doi: 10.1001/archgenpsychiatry.2008.545

16. McClure EB, Adler A, Monk CS, Cameron J, Smith S, Nelson EE, Leibenluft E, Ernst M, Pine DS. fMRI predictors of treatment outcome in pediatric anxiety disorders. Psychopharmacolgy (Berl). 2007;191(1):97-105. doi: 10.1007/s00213-006-0542-9

17. Telzer EH, Mogg K, Bradley BP, Mai X, Ernst M, Pine DS, et al. Relationship between trait anxiety, prefrontal cortex, and attention bias to angry faces in children and adolescents. Biol Psychol. 2008;79:216-22. doi: 10.1016/j.biopsycho.2008.05.004

18. Motzkin JC, Philippi CL, Wolf RC, Baskaya MK, Koenigs M. Ventromedial Prefrontal Cortex Is Critical for the Regulation of Amygdala Activity in Humans. Biol Psychiatry. 2014;77(3):276-84. doi: 10.1016/j.biopsych.2014.02.014

19. $\mathrm{Xu} \mathrm{P,} \mathrm{Gu} \mathrm{R,} \mathrm{Broster} \mathrm{LS,} \mathrm{Wu} \mathrm{R,} \mathrm{Van} \mathrm{Dam} \mathrm{NT,} \mathrm{Jiang} \mathrm{Y,} \mathrm{et} \mathrm{al.} \mathrm{Neural} \mathrm{basis} \mathrm{of}$ emotional decision making in trait anxiety. J Neurosci. 2013;33:18641-53. doi: 10.1523/JNEUROSCI.1253-13.2013

20. Guyer AE, Nelson EE, Perez-Edgar K, Hardin MG, Roberson-Nay R, Monk CS, et al. Striatal functional alteration in adolescents characterized by early childhood behavioral inhibition. J Neurosci. 2006;26:6399-405. doi: 10.1523/JNEUROSCI.0666-06.2006

21. Bar-Haim Y, Fox NA, Benson B, Guyer AE, Williams A, Nelson EE, et al. Neural correlates of reward processing in adolescents with a history of inhibited temperament. Psychol Sci. 2009;20:1009-18. doi: 10.1111/j.14679280.2009.02401.x

22. Helfinstein SM, Benson B, Perez-Edgar K, Bar-Haim Y, Detloff A, Pine DS, et al. Striatal responses to negative monetary outcomes differ between temperamentally inhibited and non-inhibited adolescents. Neuropsychologia. 2011;49:479-85. doi: 10.1016/j.neuropsychologia.2010.12.015

23. Guyer AE, Choate VR, Detloff A, Benson B, Nelson EE, Perez-Edgar K, et al. 
Striatal functional alteration during incentive anticipation in pediatric anxiety disorders. Am J Psychiatry. 2012;169:205-12. doi: 10.1176/appi.ajp.2011.11010006

24. Lago T, Davis A, Grillon C, Ernst M. Striatum on the anxiety map: Small detours into adolescence. Brain Res. 2016;1654(Pt B):177-84. doi: 10.1016/j.brainres.2016.06.006

25. Ernst M, Paulus MP. Neurobiology of decision making: A selective review from a neurocognitive and clinical perspective. Biol Psychiatry. 2005;58:597604. doi: 10.1016/j.biopsych.2005.06.004

26. Benson BE, Guyer AE, Nelson EE, Pine DS, Ernst M. Role of contingency in striatal response to incentive in adolescents with anxiety. Cogn Affect Behav Neurosci. 2014;15(1):155-68. doi: 10.3758/s13415-014-0307-6

27. Hardee JE, Benson BE, Bar-Haim Y, Mogg K, Bradley BP, Chen G, et al. Patterns of neural connectivity during an attention bias task moderate associations between early childhood temperament and internalizing symptoms in young adulthood. Biol Psychiatry. 2013;74(4):273-9. doi: 10.1016/j.biopsych.2013.01.036

28. Galvan A. Adolescent development of the reward system. Front Human Neurosci. 2010;4:6. doi: 10.3389/neuro.09.006.2010

29. Galvan A, Hare TA, Parra CE, Penn J, Voss H, Glover G, et al. Earlier development of the accumbens relative to orbitofrontal cortex might underlie risk-taking behavior in adolescents. J Neurosci. 2006; 26(25):6885-92. doi: 10.1523/JNEUROSCI.1062-06.2006

30. Galván A. The Teenage Brain: Sensitivity to Rewards. Curr Directions Psychol Sci. 2013;22:88-93. doi: 10.1177/0963721413480859

31. Rahdar A, Galván A. The cognitive and neurobiological effects of daily stress in adolescents. Neuroimage. 2014;92:267-73. doi: 10.1016/j.neuroimage.2014.02.007

32. Davidow JY, Foerde K, Galván A, Shohamy D. An Upside to Reward Sensitivity: The Hippocampus Supports Enhanced Reinforcement Learning in Adolescence. Neuron. 2016;92(1):93-9. doi: 10.1016/j.neuron.2016.08.031

33. Barkley-Levenson E, Galván A. Neural representation of expected value in the adolescent brain. Proc Natl Acad Sci U S A. 2014;111(4):1646-51. doi: 10.1073/pnas.1319762111

34. Goldenberg D, Telzer EH, Lieberman MD, Fuligni AJ, Galvan A. Greater response variability in adolescents is associated with increased white matter development. Soc Cogn Affect Neurosci. 2017;12(3):436-444. doi: 10.1093/scan/nsw132

35. de Lijster JM, Dieleman GC, Utens EMWJ, Dierckx B, Wierenga M, Verhulst FC, et al. Social and academic functioning in adolescents with anxiety disorders: A systematic review. J Affect Disord. 2018;230:108-17.

36. Merikangas KR, He JP, Burstein M, Swanson SA, Avenevoli S, Cui L, et al. Lifetime prevalence of mental disorders in U.S. adolescents: results from the National Comorbidity Survey Replication--Adolescent Supplement (NCS-A). J Am Acad Child Adolesc Psychiatry. 2010;49(10):980.

37. American Psychiatric Association. Diagnostic and statistical manual of mental 
disorders. 5th ed. Washington (D.C., US): American Psychiatric Association; 2013.

38. Zhou D, Thompson WK, Siegle G. MATLAB toolbox for functional connectivity. Neuroimage. 2009;47(4):1590-607. doi: 10.1016/j.neuroimage.2009.05.089

39. Chen CC, Kiebel SJ, Friston KJ. Dynamic causal modelling of induced responses. Neuroimage. 2008;41(4):1293-312. doi: 10.1016/j.neuroimage.2008.03.026

40. Friston K. Learning and inference in the brain. Neural Networks. 2003;16:1325-52. doi: 10.1016/j.neunet.2003.06.005

41. Siegle GJ, Ghinassi F, Thase ME. Neurobehavioral therapies in the 21st century: Summary of an emerging field and an extended example of cognitive control training for depression. Cognit Ther Res. 2007;31:235-62. doi: 10.1007/s10608-006-9118-6

42. Birmaher B, Khetarpal S, Brent D, Cully M, Balach L, Kaufman J, et al. The Screen for Child Anxiety Related Emotional Disorders (SCARED): Scale construction and psychometric characteristics. J Am Acad Child Adolesc Psychiatry. 1997;36(4):545-53. doi: 10.1097/00004583-199704000-00018

43. Hale WW III, Raaijmakers Q, Muris P, van Hoof A, Meeus W. Developmental trajectories of adolescent anxiety disorder symptoms: a 5-year prospective community study. J Am Acad Child Adolesc Psychiatry. 2008;47(5):556-64.

44. Galván A, Peris TS. Neural correlates of risky decision making in anxious youth and healthy controls. Depress Anxiety. 2014;31(7):591-8. doi: 10.1002/da.22276

45. Hanby MSR, Fales J, Nangle DW, Serwik AK, Hedrich UJ. Social Anxiety as a Predictor of Dating Aggression. J Interpers Violence. 2012; 27(10):1867-88. doi: 10.1177/0886260511431438

46. Kashdan TB, Elhai JD, Breen WE. Social anxiety and disinhibition: An analysis of curiosity and social rank appraisals, approach-avoidance conflicts, and disruptive risk-taking behavior. J Anxiety Disord. 2008; 22(6):925-39. doi: 10.1016/j.janxdis.2007.09.009

47. Kashdan TB, Hofmann SG. The high-novelty-seeking, impulsive subtype of generalized social anxiety disorder. Depress Anxiety. 2008;25(6):535-41. doi: 10.1002/da.20382

48. Kashdan TB, McKnight PE. The darker side of social anxiety: When aggressive impulsivity prevails over shy inhibition. Curr Dir Psychol Sci. 2010;19(1):4750. doi: 10.1177/0963721409359280

49. Kashdan TB, McKnight PE, Richey JA, Hofmann SG. When social anxiety disorder co-exists with risk-prone, approach behavior: Investigating a neglected, meaningful subset of people in the National Comorbidity SurveyReplication. Behav Res Ther. 2009;47(7):559-68. doi: 10.1016/j.brat.2009.03.010

50. Rounds JS, Beck JG, Grant DMM. Is the delay discounting paradigm useful in understanding social anxiety? Behav Res Ther. 2007;45(4):729-35. doi: 10.1016/j.brat.2006.06.007

51. Schneier FR, Foose TE, Hasin DS, Heimberg RG, Liu SM, Grant BF, et al. Social anxiety disorder and alcohol use disorder co-morbidity in the national epidemiologic survey on alcohol and related conditions. Psychol Med. 
2010;40(6): 977-88. doi: 10.1017/S0033291709991231

52. Silverman WK, Albano AM. The anxiety disorders interview schedule for DSM-IV: Child and parent versions. San Antonio (TX, US): Graywind; 1996.

53. Stallings P, March JS. Chapter: Assessment. Anxiety disorders in children and adolescents. New York (US): Guilford Press; 1995. p. 125-47.

54. Silverman WK, Nelles WB. The Anxiety Disorders Interview Schedule for Children. J Am Acad Child Adolesc Psychiatry. 1988;27(6):772-8. doi: 10.1097/00004583-198811000-00019

55. Rozenman M, Amir N, Weersing VR. Performance-based interpretation bias in clinically anxious youths: Relationships with attention, anxiety, and negative cognition. Behav Ther. 2014;45(5):594-605. doi: 10.1016/j.beth.2014.03.009

56. Levin IP, Hart SS. Risk Preferences in Young Children: Early Evidence of Individual Differences in Reaction to Potential Gains and Losses. J Behav Decis Mak. 2003;16(5):397-413. doi: 10.1002/bdm.453

57. Quinn EP, Brandon TH, Copeland AL. Is task persistence related to smoking and substance abuse? The application of learned industriousness theory to addictive behaviors. Exp Clin Psychopharmacol. 1996;4(2):186-90. doi: 10.1037/1064-1297.4.2.186

58. Ehrlich KB, Cassidy J, Gorka SM, Lejuez CW, Daughters SB. Adolescent friendships in the context of dual risk: The roles of low adolescent distress tolerance and harsh parental response to adolescent distress. Emotion. 2013;13(5):843-51. doi: 10.1037/a0032587

How to cite this article:

Galván A, Peris TS. The Development of Anxiety in Youth Study (DAYS): A Prospective Study of Trajectories of Brain Maturation among Youth at Risk for Anxiety. J Psychiatry Brain Sci. 2020;5:e200025. 